
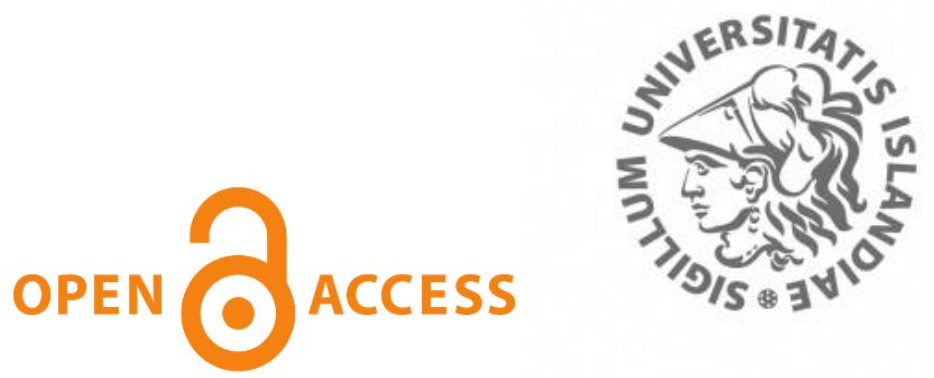

\title{
Opin vísindi
}

This is not the published version of the article / Petta er ekki útgefna útgáfa greinarinnar

Author(s)/Höf.: $\quad$ O. Bălan, A. Moldoveanu, F. Moldoveanu, H. Nagy, G. Wersényi and R. Unnthorsson.

Title/Titill: Improving the Audio Game-Playing Performances of People with Visual Impairments Through Multimodal Training

Year/Útgáfuár: $\quad 2017$

Version/Útgáfa: $\quad$ Post-print / Lokagerð höfundar

\section{Please cite the original version:}

\section{Vinsamlega vísið til útgefnu greinarinnar:}

O. Bălan, A. Moldoveanu, F. Moldoveanu, H. Nagy, G. Wersényi and R. Unnthorsson. (2017). Improving the Audio Game-Playing Performances of People with Visual Impairments Through Multimodal Training. Journal of Visual Impairment \& Blindness, $111(2), 148-164$

Rights/Réttur: (C)2017 AFB, All Rights Reserved 


\title{
Improving the audio game playing performances of the people with visual impairment through multimodal training
}

\author{
Oana Bălan ${ }^{1}$, Alin Moldoveanu ${ }^{1}$, Florica Moldoveanu ${ }^{1}$, Hunor Nagy $^{2}$, György Wersényi ${ }^{2}$ \\ Rúnar Unnpórsson ${ }^{3}$ \\ 1 - University POLITEHNICA of Bucharest, Faculty of Automatic Control and Computers, Splaiul \\ Independentei, 313, 060042, Bucharest, Romania \\ 2 - Széchenyi István University, Department of Telecommunications, H-9026, Győr, Hungary \\ 3 - University of Iceland, \\ School of Engineering and Natural Sciences - Faculty of Industrial Engineering, Mechanical Engineering and \\ Computer Science, VR-2 /V02-237, Reykjavik, Iceland
}

\section{AUTHOR NOTE}

Correspondence: Oana Bălan, University POLITEHNICA of Bucharest, Faculty of Automatic Control and Computers, Splaiul Independentei, 313, 060042, Bucharest, Romania, email: oanab_2005@yahoo.com / oana.balan@cs.pub.ro 


\begin{abstract}
Introduction: As the number of people with visual impairments is continuously increasing, rehabilitation and engineering researchers have identified the need to design sensory substitution devices that would offer assistance and guidance to the blind users for performing navigational tasks. Auditory and haptic cues have been shown to be an effective approach towards creating a rich spatial representation of the environment, so that they are considered for being included into the development of assistive tools that would enable the people with visual impairments to acquire knowledge of the surrounding space in a way close to the visual based perception of the normally sighted individuals. However, achieving efficiency through a sensory substitution device requires extensive training for the blind users to learn how to process the artificial auditory cues and convert them into spatial information.
\end{abstract}

Methods: Considering all the potential advantages which game based learning can provide, we propose a new method for training sound localization and virtual navigational skills of the people with visual impairments in a 3D audio game with hierarchical levels of difficulty. The training procedure is focused on a multimodal (auditory and haptic) learning approach in which the subjects have been asked to listen to 3D sounds while simultaneously perceiving a series of vibrations on a haptic headband, corresponding to the direction of the sound source in space.

Results: The results we obtained in a sound localization experiment with 10 people suffering from visual impairments showed that the proposed training strategy resulted in significant improvements in the auditory performance and navigation skills of the subjects, thus ensuring behavioral gains in the spatial perception of the environment.

Keywords: Sensory Substitution, Sound Localization, Game Based Training, Audio Games, Virtual Auditory Environment, HRTF. 


\section{Introduction}

More than 285 million people worldwide suffer from a certain degree of visual impairment, of which 40 million are totally blind (World Health Organization statistics, 2015). Some people are affected by the lack of social involvement, a sedentary, home-confined lifestyle, as well as the inability to take care of themselves (Constâncio, 2010). Effective assistive solutions can improve mobility, cardinality and social integration. A detailed representation of the environment can improve the navigational skills of the people suffering from visual impairments (Afonso et al, 2010). In compensation for the lack of sight, the blind individuals must rely more on alternative sensory modalities, such as hearing and touch. This connected to the idea of integrating auditory and haptic (tactile and kinesthetic) stimuli into the development of assistive devices in order to create and deliver a thorough representation of the surrounding space (Jaijongrak et al, 2011).

The purpose of this paper is to investigate the improvements occurring in the sound localization performance and elementary virtual environment navigational skills of the people with visual impairments as a result of training. Training the sound localization skills of the blind subjects leads to improved spatial auditory performances which will be reflected by more accurate representation of the environment and enhanced navigational skills. We propose a realistic, immersive, feedback-rich, user-centered and motivational ludic-based approach to training through virtual games. This approach will enable the people with visual impairments to develop sound localization skills, acquire situational knowledge from audio cues, collect directional information and develop auditory-based spatial understanding and virtual navigational skills in a dynamic and interactive way. 


\section{Training sound localization through audio games}

Many experiments have demonstrated that audio game based training can be a reliable strategy for improving spatial auditory resolution in virtual environments (Sanchez et al, 2010; Zahorik et al, 2006; Bălan et al, 2015b).

In the experiment presented in (Blum et al, 2004), a proprioceptive feedback method was used to help the blindfolded subjects manipulate sound sources located at their hand position (which has the advantage of being egocentric for the user). In the training session (designed as a gamelike scenario), the subjects were required to search for animal sounds hidden around them. Using a positional hand tracked ball (the sound being spatialized at the ball center), they were required to search the perceived direction of the sound. When the subject identified the correct location, the pink noise used as primary sound stimulus was replaced by a sound that was similar to that produced by an animal and the next direction was generated having as reference point the previous sound source location. In this way, the subjects were provided with continuous auditory feedback and could explore their entire sphere of perception and action. The experimental results concluded that the angular error was reduced with 6.1 degrees.

In (Honda et al, 2007), the transfer effects of playing an audio game were analyzed in a sound localization experiment in which two groups of subjects participated: a group that used generic Head Related Transfer Functions (HRTFs) and another group that used individualized HRTFs. The HRTFs are a measure of the sound transformation from the source to the listener's ears (Spagnol et al, 2013; Meshram et al, 2014) that are highly dependent on the anatomical characteristics of the listener's body (size and shape of the pinna, head and torso). Computer generated 3D binaural sounds are made by convolving a monaural signal with the corresponding 
HRTFs of a particular direction in space. The training method consisted of an audio game entitled Bee Bee Beat, in which the sound of a honeybee appeared at an arbitrary direction in space and the listener was required to use a plastic hammer to hit the noise. After the listener identified the location of the target, he received immediate vibration feedback from the hammer. The trained group was asked to play the game for 30 minutes per day, for a period of 2 weeks. Consequently to training, the sound localization accuracy of the subjects increased with $20 \%$.

The Audio Doom game (Lessard et al, 1998) aimed to enhance the cognitive abilities and virtual navigational skills of the blind children. The players needed to explore a virtual environment which consisted of a 3D labyrinth of wall and corridors, avoid monsters and find the exit to the next level while listening to auditory cues, such as the sound of footsteps or that of a door opening. Seven blind children who played the game succeeded to improve their spatial cognitive skills. The transfer of learning into real-world situations was demonstrated by the ability to recreate the route they travelled in the game using Lego blocks (Bălan et al, 2015a).

In Pyvox (Gaudy et al, 2009), the virtual environment was represented by a tower with 70 floors, corresponding to the 70 levels of the game. The player was required to find the exit for each floor, without hitting the walls. The sonification approach encoded the walls with the sound of an unpleasant noise, while to the exit of each level it had been assigned a stereo rendering that varied in pitch according to the distance to the current position of the player. An experiment performed with two groups of blind people showed that they understood the principles of the game and succeeded to complete at least 3 levels of it (Bălan et al, 2015a).

In BlindSide (Blind Side; Reinhard, 2014; Parker, 2013), the virtual environment consisted of the settings of a building and a city, while the sonification technique included 3D binaural and 
IMPROVING THE AUDIO GAME PLAYING PERFORMANCES OF THE VISUALLY IMPAIRED PEOPLE THROUGH MULTIMODAL TRAINING

pre-recorded sounds, narration sequences and many realistic effects. In an experiment which included both sighted people and persons with visual impairments, the results showed that the latter were able to play the game more accurately and to finish it earlier than their sighted counterparts (Bălan et al, 2014).

In (Balan et al, 2015c), the sound localization performance of nine subjects with visual impairments was assessed before and after a series of haptic-auditory training procedures aimed to enhance the perception of 3D sounds. The test results showed that the subjects improved their sound localization performance and reduced the incidence of angular precision and reversal errors. Our experiment was composed of a pre-test session, a training session (based on hapticauditory perceptual feedback, aimed to help the subjects to get used to the perception of 3D sounds delivered via headphones) and a post-test session (identical with the pre-test session), which had the purpose of assessing the degree of acoustic spatial resolution improvement achieved as a result of the perceptual training procedure.

\section{Method}

\section{Overview}

This study comprises the assessment of the sound localization skills of 10 subjects with visual impairments who were required to play an audio game with hierarchical levels of difficulty. In the game, the players had to identify the location of several hidden auditory targets while trying to avoid the obstacles standing in their way. The experiment was composed of a pre-test session (in which the subjects were asked to play the game twice, for two different sets of levels), a training session (aimed at helping the individuals with visual impairments to adapt to the perception of 3D sounds through multimodal interaction - both auditory and haptic) and a post- 
IMPROVING THE AUDIO GAME PLAYING PERFORMANCES OF THE VISUALLY IMPAIRED PEOPLE THROUGH MULTIMODAL TRAINING

test session (similar in structure and difficulty to the pre-test), in which the degree of sound localization improvement following training was evaluated.

\section{Participants}

Ten individuals with visual impairments (5 women and 5 men, aged 27-63, mean age=43, with a percent of residual vision ranging between $0 \%$ and $15 \%$ participated in our experiment. Residual vision represents the degree of remaining vision, measured in percent. Prior to the experiment, each subject declared his or her level of residual vision, determined by the ophthalmologist. The subjects' visual acuity ranged from 20/200 to 20/1000, visual field of 20 degrees or less. Two of the subjects were congenitally blind, one was congenitally visually impaired (with $10 \%$ vision), while the others were late-onset visually impaired. The experiments followed the tenets of the World Medical Association Declaration of Helsinki on Ethical Principles for Medical Research Involving Human Subjects. The Blind People Association from Györ, Hungary approved the experiments and written informed consent was obtained from all subjects.

\section{Sound stimuli}

The auditory stimuli were continuous 3D binaural sounds synthesized with non-individualized HRTFs taken from the Massachusetts Institute of Technology (MIT) dataset (MIT HRTF Database), auditory icons (sounds that create an analogy with real-world events and situations) and earcons (abstract, symbolic sounds used to facilitate the players' navigation through the complex content of the game) (Csapó \& Wersényi, 2013). The location of a hidden auditory target was sonified using a combination of white and pink noise in varying proportions (both noises could be perceived simultaneously), so that at 0 degrees to the front the listener hears only 
IMPROVING THE AUDIO GAME PLAYING PERFORMANCES OF THE VISUALLY IMPAIRED PEOPLE THROUGH MULTIMODAL TRAINING

white noise and at 180 degrees to the back the listener hears only pink noise. In the right hemifield, the proportion of white noise decreased and that of pink noise increased, reaching equal levels at 90 degrees (Figure 1). On the other hand, in the left hemifield, the percent of pink noise decreased and that of white noise increased, reaching equal levels at 270 degrees. The white noise presents a constant power spectral density (equal power per Hertz) (Wikipedia, Power Spectral Density), which means that it contains every frequency within the range of human hearing (generally from 20 hertz to $20 \mathrm{kHz}$ ) in equal amounts (Wikipedia, White noise). The pink noise is a random signal with a power spectral density that is inversely proportional to the frequency of the signal, so that each octave contains an equal amount of noise energy (Wikipedia, Pink noise). The formula for calculating the proportion of white and pink noise for a given direction in space in our experiment is the following (Bălan et al, 2015c):

$$
\begin{gathered}
\text { pink }=\text { angle } / 180 ; \text { white }=1-\text { pink; }(0 \leq \text { angle } \leq 180) \\
\text { white }=(\text { angle-180 }) / 180 ; \text { pink }=1-\text { white } ;(180<\text { angle }<360)
\end{gathered}
$$

The purpose for this combination of white and pink noise was to reduce the incidence of front-back confusions by enabling the listener to differentiate the direction of source, based on the spectral profile of the sound.

The location of the obstacles was encoded using an alarm sound that was spatialized using the non-individualized HRTFs from the MIT database, so that the players could identify the direction of both targets and obstacles. The auditory icons used were the alarm sounds aimed at raising the player's awareness in what concerns approaching the obstacles and the sound of a 
IMPROVING THE AUDIO GAME PLAYING PERFORMANCES OF THE VISUALLY IMPAIRED PEOPLE THROUGH MULTIMODAL TRAINING

crash (or accident) when he ran into an obstacle. Earcons have been represented by the sound of a click when the player succeeded to identify the position of a target and a bell ringing that announced the end of the game. When the subjects approached targets or obstacles, the perceived sound intensity increased and when they receded from the objects the intensity decreased.

\section{Procedure}

In the pre-test session, the sound localization performance of the subjects with visual impairments has been evaluated in an audio game where the subjects were required to identify the location of several hidden auditory targets while trying to avoid blocking obstacles (Figure 2). The game had 10 levels of difficulty designed in the $2 \mathrm{D}$ space, with varying numbers of targets and obstacles, as it follows:

- Level 1: 1 target and 1 obstacle

- Level 2: 1 target and 2 obstacles

- Level 3: 2 targets and 2 obstacles

- Level 4: 2 targets and 3 obstacles

- Level 5: 3 targets and 3 obstacles

- Level 6: 3 targets and 4 obstacles

- Level 7: 4 targets and 4 obstacles

- Level 8: 4 targets and 5 obstacles

- Level 9: 5 targets and 5 obstacles

- Level 10: 5 targets and 5 obstacles 
At each level, the target sound sources were positioned starting from the bottom border of the playing window to the top of it, so that once the player discovered one target, the player was required to look for the next ones above, reducing thus the searching area and further preventing the occurrence of front-back localization misjudgments. Only one sound target was active (could be heard) at a moment of time. Once the position of the current target was identified, it consequently became inactive and the next target (in ascending order, from the bottom side of the playing window to the top of it) became audible. An obstacle became audible when the player was within a range of 150 pixels from the obstacle. When two obstacles were less than 150 pixels away from the player two sound stimuli with different directional cues, corresponding to each obstacle, were presented.

The players were required to navigate freely, using the mouse or touchpad movement as interaction modality. Prior to the experiment, the subjects were instructed to use the mouse or the touchpad, according to preference or prior experience. As they were modifying their position in respect with the active target and the surrounding obstacles, they could perceive changes in both the spectral content of the sound, its intensity, and its localization simulated with HRTFs. During the experiment, the subjects with low vision were blindfolded.

During the pre-test session, the subjects were required to play the game twice, with two different sets of levels (set 1 and set 2) (Figures 2 and 3). Before the pre-test, the subjects were presented the purpose of the game and the main auditory cues used in the sonification strategy. Moreover, in order to allow them to get familiar with the aim of the game and with the perception of $3 \mathrm{D}$ sounds, the subjects were allowed to practice playing the game as long as they considered it was necessary prior to the start of the tests. Usually, most of the subjects played the 
IMPROVING THE AUDIO GAME PLAYING PERFORMANCES OF THE VISUALLY IMPAIRED PEOPLE THROUGH MULTIMODAL TRAINING

game once (10 levels), for an average time of 10 minutes, until they got accustomed to the sonification approach and the aim of the game.

The studied parameters were:

- P1: The ratio of the distance travelled by the player (from the starting position until he discovered the location of the current target) to the minimum possible distance (the Euclidean distance between the starting point and the position of the current target). For the first target of any level, the starting position was the center of the bottom border of the playing window. For the other targets, the starting position was the location of the previously identified target.

- P2: The percent of correct travel decisions, defined as movements effectuated towards the sound source (minimizing the distance between the user's virtual location and the position of the target).

- P3: The average level completion time (in seconds).

- P4: The average number of obstacle hits.

During the training session, the subjects were provided with haptic feedback which conveyed the direction of the sound source. Thus, the subjects wore stereophonic headphones and a haptic headband which contained 24 vibration motors (evenly distributed around the head) that transmitted vibrations corresponding to the direction of the sound source in space.

The haptic system was composed of several parts (Figure 4):

- The USB Wireless Gateway Device (UWGD);

- Haptic Actuator Device (HAD) that effectively controlled the haptic actuators (Eccentric Rotating Mass ERM motors); 
IMPROVING THE AUDIO GAME PLAYING PERFORMANCES OF THE VISUALLY IMPAIRED PEOPLE THROUGH MULTIMODAL TRAINING

- 24 vibration motors, fixed along a stick in order to allow easy handling. The motors were evenly distributed around the head of the subject. As the head size and shape varied significantly among subjects, the experimenter personally checked and ensured accurate placement of the vibration motors at their corresponding directions on the haptic belt for each listener apart.

The training session took place in two consecutive days. Both days, the subjects went through three training blocks - each with duration of 3 minutes - where the subjects were required to listen to a series of 24 sounds (emulated in clockwise order from 0 to 345 degrees) and then to randomly generated auditory stimuli with duration of 4 seconds. The sounds used were a combination of white and pink noise in varying proportions - the same encoding used for the game described in previous section. Each sound stimulus perceived through the headphones was accompanied by a train of 4 vibrations on the haptic headband (one vibration per second) corresponding to the direction of the sound in space. The purpose of the training session was to help the subjects with visual impairments to get used to the perception of 3D sounds and to create an effective crossmodal association (haptic and auditory) that would help the subjects to easily identify the direction of the 3D sounds. The high resolution of the haptic headband (the vibration motors were evenly distributed around the subject's head - resulting in a $15^{\circ}$ angle between the motors) allowed for accurate training, a strategy aiming to offer a more accurate spatial perception of the environment (Figure 5). 
IMPROVING THE AUDIO GAME PLAYING PERFORMANCES OF THE VISUALLY IMPAIRED PEOPLE THROUGH MULTIMODAL TRAINING

The post-test session, that took place one day after training, has been carried out in exactly the same conditions as the pre-test session, using the audio-based game. The goal of the post-test session was to assess the level of sound localization and virtual navigational skills improvement achieved after training.

\section{Software tools}

The game presented a functionality that allowed saving the current gameplay records (the records of the player's movements on the computer screen) into a log file that can be uploaded later on for analyzing and visualizing the results.

For designing each level (number and location of targets and obstacles), a tool called Game Editor was developed. It allowed the experimenter to set the difficulty of the levels by manually placing targets and obstacles inside the playing window (Figure 6).

The Game Analyzer tool allowed visualization, synchronized playback of the subjects' game performance, basic statistical analysis (mean values for all the 4 studied parameters) - for each level and for each target. The segments that were colored in green (black in the colored version of the paper) represented good movements (getting closer to the target), while those painted in red (black in the colored version of the paper) represented incorrect travel decisions (getting farther away from the target). The "Export all data" button allowed the results to be exported to an Excel file for further analysis (Figure 7). Overall, the Game Analyzer tool proved to be an excellent analysis solution, which, besides direct observation of the subjects during the experiment, facilitated better understanding of the in-game behavior and the issues which the subjects were confronted with. 
Seven of our subjects participated in a sound localization experiment that took place 2 months before the current one (Bălan et al, 2015c). In the previous experiment, the sound localization accuracy of 9 people with visual impairments was tested before and after a training session based on auditory and haptic feedback. In both the pre-test and the post-test sessions, the subjects were required to identify the position of a sound source by freely navigating from the starting position (the center of a circle of 150 pixels radius) to the actual location of the target (which was randomly generated on the margin of the circle) (Figure 8). The sound stimuli was a combination of white and pink noise in varying proportions, according to the direction of the sound source in space (previously described in this paper) and a "ding" type signal with a narrower spectral profile. In the training session, the subjects received auditory and haptic feedback about the direction of the sound source in space through the haptic belt they were required to wear on the head. In that case, the vibration motors were placed at 30 degrees difference around the head, offering a lower spatial resolution than in the current experiment. The results obtained demonstrated a more accurate sound localization performance and enhanced localization skills in the post-test session of the experiment, notably for the trials where the white/pink noise combination has been employed as primary auditory cue.

\section{Results}

Table 1 briefly presents a statistical overview of the results obtained by the subjects (mean, SD - standard deviation, minimum and maximum value) in both the pre-test and post-test sessions of the experiment for parameters P1-P4 (Figure 9, Figure10, Figure 11, Figure 12). Table 2 presents the raw results of the experiment. 
More than $80 \%$ of the subjects succeeded to enhance their sound localization and navigational performance in the post-test session of the experiment (Table 3). All the participants recorded a higher rate of correct travel decisions towards the target sound source.

Table 4 presents the mean results for all the four parameters, for both sets of levels, in the pre-test and post-test sessions of the experiment.

In what concerns the results obtained for the second set of levels, the results obtained in both the pre-test and in the post-test sessions are higher than those recorded for the first set. Thus, the mean value of parameter P1 decreased with $14 \%$ (from 3.1 to 2.7 - although the results are not statistically significant), the mean rate of correct travel decisions increased with $7.7 \%$ (from $74.3 \%$ to $82.1 \%$ - the differences between the performance in the pre-test and post-test sessions are statistically significant in an ANOVA test at $\mathrm{p} \leq 0.05$ and in a Student $\mathrm{t}$-test where $\mathrm{t}=3.4$ at $\mathrm{p} \leq 0.05$ ), the level completion time reduced with $20.4 \%$ (from 32.5 seconds to 25.9 seconds in the post-test session - the differences are not statistically significant), while the mean rate of obstacle hits per level decreased with $14.2 \%$ (from 0.30 to 0.26 - the results are not statistically significant) (Table 5).

The results presented in Table 6 show that the mean rate of parameters P1 and P2 is higher in the current experiment than in the one performed two months prior to this one (Bălan et al, 2015c), demonstrating the persistence of the spatial auditory adaptation of the human hearing system and its continuous improvement in time. 
IMPROVING THE AUDIO GAME PLAYING PERFORMANCES OF THE VISUALLY IMPAIRED PEOPLE THROUGH MULTIMODAL TRAINING

The mean rate of parameter P1 improved with $43.1 \%(\mathrm{t}=1.68, \mathrm{p} \leq 0.1)$ and that of parameter $\mathrm{P} 2$ with $5.64 \%$ (the results are statistically in an ANOVA test at $\mathrm{p} \leq 0.1$ and in a Student $\mathrm{t}$-test where $\mathrm{t}=2.08$ at $\mathrm{p} \leq 0.1)$ between the post-test session of the previous experiment and the pre-test session of the current one. Also, $85 \%$ (6 out of the 7 subjects) recorded more accurate sound localization abilities in the pre-test session of the current experiment that in the post-test phase of the previous one, fact that can be explained by their familiarity with the tasks and with the sound stimuli - the perception of directionality conveyed by the 3D sounds, the spectral characteristics of the white and pink noise and the continuous change in sound intensity that is inversely proportional to the distance between the listener and the sound source.

The final results demonstrate significant improvements in the post-test session of the present experiment, surpassing those obtained in the previous experiment with $57.4 \%$ (the results are statistically significant in an ANOVA test at $\mathrm{p} \leq 0.1$ ) for parameter P1 (from 6.68 to 2.84 ) and with $13.8 \%$ (the differences are statistically significant in an ANOVA test at $\mathrm{p} \leq 0.05$ and in a Student t-test for dependent means where $\mathrm{t}=3.5$ at $\mathrm{p} \leq 0.5$ ) for $\mathrm{P} 2$ (from $66.15 \%$ to $80 \%$ ).

Moreover, the players who participated in the previous experiment were more proficient in accomplishing the virtual navigational and sound localization tasks required in the current game than their inexperienced counterparts. At the same time, the experienced users recorded a higher improvement in the post-test session of the current experiment (compared to the pre-test session), increasing their percentage of correct travel decisions with $8.3 \%$ (from $71.7 \%$ to $80 \%$ - the results are statistically significant in an ANOVA test at $p \leq 0.05$ and in a Student $t$-test where $\mathrm{t}=3.66$ at $\mathrm{p} \leq 0.05)$. 


\section{Discussion}

The results show that using the proposed multimodal (auditory and haptic) training strategythe people with visual impairments succeeded to achieve a rapid improvement of their sound localization abilities and auditory-based virtual navigational skills. Extensive gameplay in the pre-test and post-test sessions of the experiment provided an important contribution to the improvement of the sound localization accuracy and virtual navigational skills, as it enabled the subjects to gain experience with the game.

The training procedure helped the subjects to adapt to stimulus conditions that present a mismatch between the spectral cues and the sound direction, such as the use of 3D binaural sounds synthesized with non-individualized HRTFs in virtual auditory environments. Moreover, the perceptual training enabled the subjects to map the virtual settings and to perform simple navigational tasks (target localization, obstacle avoidance and effective gameplay).

The subjects based their game playing strategy on the perception of both the directional binaural sounds which gave clear clues about the location of both targets and obstacles in space, and also on the perception of continuous changes in sound intensity. The improvement in sound localization accuracy is explained by the perceptual training method that used broadband noises containing more spectral cues for the learning/retrieving process. Moreover, even if these stimuli are not natural, they are effective for training due to their enhanced externalization features (Mendonça et al, 2013). Another argument that supports the efficiency of the training session is that the subjects were only trained using 24 virtual sound source positions, whereas error reductions and improved spatial perception have been recorded for many other stimuli directions, including the untrained positions. One of the most remarkable results reported in this study are 
IMPROVING THE AUDIO GAME PLAYING PERFORMANCES OF THE VISUALLY IMPAIRED PEOPLE THROUGH MULTIMODAL TRAINING

the long-lasting effects of the training sessions performed in the previous experiment. However, the results of the pre-test session of the current experiment are more accurate than those recorded in the post-test phase of the previous one, demonstrating that the spatial auditory remapping is a continuous process and that a new, solid and persistent head model is developed for localizing altered sound cues, in accordance to Hofman's theory (Hofman et al, 1998; Mendonça et al, 2013).

To sum up, the ratio of the total distance travelled by the listeners to the minimum possible distance decreased by $27 \%$, the rate of correct travel decisions towards identifying the location of the target sound sources improved with approximately $7 \%$, the mean level completion time reduced with $33 \%$, while the number of obstacle hits per level decreased by a half in the post-test session of the experiment.

As the results obtained in both the pre-test and post-test sessions of the current experiment are higher than those achieved in the previous one, we shed some light on the fact that the human auditory system is able to continuously improve its sound localization abilities and that experience-driven learning plays a fundamental role in enhancing the navigational and spatial cognitive skills of the individuals with visual impairments.

\section{Conclusions}

The experimental results of this research demonstrated that the people with visual impairments are able to perform route-navigational tasks (such as searching for auditory targets or avoiding obstacles) in virtual reality environments using 3D binaural sounds as the only means for navigation. Moreover, the brief multimodal (auditory and haptic) training session helped the subjects to adapt to altered hearing conditions (such as the use of 3D sounds filtered 
IMPROVING THE AUDIO GAME PLAYING PERFORMANCES OF THE VISUALLY IMPAIRED PEOPLE THROUGH MULTIMODAL TRAINING

with non-individualized HRTFs) had a contribution to creating an association between the auditory stimuli perceived in the headphones and the vibrations corresponding to the direction of the sound source on the haptic belt.

A limitation to the study can be considered the prior experience of the 7 subjects who participated in a similar sound localization experiment that took place 2 months before this one. Thus, they were familiar with the procedure, the haptic device and the perception of 3D binaural sounds. Another limitation is the small sample size of subjects that led to statistically insignificant results for some parameters of the study.

This study has applicability for the Sound of Vision project (Sound of Vision website), which is a European research project which intends to develop an assistive device dedicated to the people suffering from visual impairments. The system will encode the environmental information into auditory and haptic stimuli. It will not use traditional supra-aural headphones, but custom-designed bone conduction or multi-speakers, in order to prevent the user from losing the ability to hear environmental information. The results of the current study will be used for the development of a training strategy based on multimodal perceptual feedback and virtual reality that will enable the users of the Sound of Vision device to become familiar with the system before using it in the real-world environment.

Furthermore, as the audio games are not restricted to the blind community only, we consider that this game can be played by the sighted people who want to try an alternative to the video games, entertain and train their sound localization skills at the same time. 
IMPROVING THE AUDIO GAME PLAYING PERFORMANCES OF THE VISUALLY IMPAIRED PEOPLE

THROUGH MULTIMODAL TRAINING

\section{Acknowledgement}

The work has been funded by the Sectoral Operational Programme Human Resources Development 2007-2013 of the Ministry of European Funds through the Financial Agreement POSDRU/159/1.5/S/132395. This project has received funding from the European Union's Horizon 2020 research and innovation programme under grant agreement No 643636 "Sound of Vision".

\section{REFERENCES}

Afonso, A., Blum, A., Katz, B. F. G., Tarroux, P., Borst, G., \& Denis, M. (2010). Structural properties of spatial representations in blind people: Scanning images constructed from haptic exploration or from locomotion in a 3-D audio virtual environment. Memory \& Cognition, 38, 591-604.

Bălan, O., Moldoveanu, A., \& Moldoveanu, F. (2015). Navigational Audio Games: An Effective Approach Towards Improving Spatial Contextual Learning for Blind People. The International Journal on Disability and Human Development, 14(2), 109-118.

Bălan, O., Moldoveanu, A., Moldoveanu, F., \& Dascălu, M.I. (2014). Navigational 3D AudioBased Game- Training Towards Rich Auditory Spatial Representation of the Environment. Proceedings of the 18th International Conference on System Theory, Control and Computing, Sinaia, Romania, October 17-19, 2014, 688-693, ISBN 978-1-4799-4602-0.

Bălan, O., Moldoveanu, A., Moldoveanu, F., \& Negoi, I. (2015). The Role of Perceptual Feedback Training on Sound Localization Accuracy in Audio Experiments. Proceedings of The 11th International Scientific Conference eLearning and software for Education, Bucharest, April 23-24, 2015.

Bălan, O., Moldoveanu, A., Nagy, H., Wersényi, Gy., Botezatu, N., Stan, A., \& Lupu, R.G. (2015). Haptic-Auditory Perceptual Feedback Based Training for Improving the Spatial Acoustic Resolution of the People with visual impairment. The 21st International Conference on Auditory Display (ICAD-2015), July 8-10, 2015, Graz, Austria.

Blum, A., Katz, B. F. G., \& Warusfel, O. (2004). Eliciting adaptation to non-individual HRTF spectral cues with multi-modal training. Proceedings of Joint Meeting of the German and the French Acoustical Societies (CFA/DAGA '04), Strasboug, France. 1225-1226. 
Constâncio, J.L.L. (2010). The visually impaired: involvement in physical activity and sport. Revista Digital - Buenos Aires, 146. Available at: http://www.efdeportes.com/efd146/thevisually-impaired-in-sport.htm.

Csapó, Á., \& Wersényi, Gy. (2013). Overview of auditory representations in human-machine interfaces, Journal ACM Computing Surveys (CSUR), 46(2).

Gaudy, T., Natkin, S., \& Archambault, D. (2009). Pyvox 2: An Audio Game Accessible To Visually Impaired People Playable Without Visual Nor Verbal Instructions. Transaction on Edutainment II, LNCS 5660, 176-186.

Hofman, P.M., van Riswick, J.G.A, \& van Opstal, A.J. (1998). Relearning sound localization with new ears. Nat.Neurosci. 1, 417-421. doi:10.1038/1633.

Honda, A., Shibata, H., Gyoba, J., Saitou, K., Iwaya, Y., \& Suzuki, Y. (2007). Transfer effects on sound localization performances from playing a virtual three-dimensional auditory game. Appl Acoustics, 68(8), 885-896.

Jaijongrak, V., Kumazawa, I., \& Thiemjarus, S. (2011). A haptic and auditory assistive user interface: Helping the blinds on their computer operations,". The 12th IEEE International Conference on Rehabilitation Robotics, Jun. 2011, 154-159, eTH Zurich, Switzerland.

Lessard, N., Pare, M., Lepore, F., \& Lassonde, M. (1998). Early-blind human subjects localize sound sources better than sighted subjects. Nature, 395, 278-80.

Mendonça, C., Campos, G., Dias, P., \& Santos, J.A. (2013). Learning Auditory Space: Generalization and Long-Term Effects. PLOS ONE 8(10). doi:10.1371/journal.pone.0077900.

Meshram, A., Mehra, R., Yang, H., Dunn, E., Frahm, J.M., \& Manocha, D. (2014). P-HRTF: Efficient personalized HRTF computation for high-fidelity spatial sound. International Symposium on Mixed and Augmented Reality.

Sanchez, J., Saenz, M., Pascual-Leone, A., \& Merabet, L. (2010). Navigation for the blind through audio-based virtual environments. Proceedings of the CHI Conference, Atlanta, Georgia, USA, 2010.

Spagnol, S., Geronazzo, M., Rocchesso, D., Avanzini, F. (2013). Extraction of Pinna Features for Customized Binaural Audio Delivery on Mobile Devices. Proceedings of International Conference on Advances in Mobile Computing \& Multimedia, Vienna, Austria, 2-4 December 2013. 
Zahorik, P., Bangayan, P., Sundareswaran, V., Wang, K., \& Tam, C. (2006). Perceptual recalibration in human sound localization: Learning to remediate front-back reversals. $J$ Acoust Soc Am 120, 343-359. doi: 10.1121/1.2208429.

Blind Side. http://michaeltastolfi.com/\#/blindside. 2015.

MIT HRTF Database. http://sound.media.mit.edu/resources/KEMAR.html. 2015.

Parker, L. (2013). A Video Game that You Can't Even See. http://www.newyorker.com/tech/elements/a-video-game-that-you-cant-even-see. 2013.

Reinhard, B (2014). A Sound Solution: History of Audio Games For The Visually Impaired. http://artistryingames.com/sound-solution-history-audio-games-visually-impaired/. 2014.

Sound of Vision. http://www.soundofvision.net/. 2015.

Wikipedia, Pink noise. https://en.wikipedia.org/wiki/Pink_noise. 2016

Wikipedia, Power Spectral Density.

https://en.wikipedia.org/wiki/Spectral_density\#Power_spectral_density. 2016

Wikipedia, White noise. https://en.wikipedia.org/wiki/White_noise. 2016

World Health Organization statistics. http://www.who.int/mediacentre/factsheets/fs282/en/. 2015. 